\title{
Trocas alimentares entre bebês irmãos de autistas e suas mães: risco ou recurso?*1
}

\author{
Nathalia Teixeira Caldas Campana*2 \\ Rogério Lerner*3
}

Partindo da importância que a ingestão de alimentos tem para a construção da realidade interna do bebê, este trabalho propõe discutir a relação que mães de um primeiro filho diagnosticado como autista estabelecem com seus filhos mais novos durante a alimentação. Serão apresentadas vinhetas clínicas que enfatizam as noções de necessidade e demanda, aspectos da relação entre os pais e a criança e os efeitos terapêuticos que as entrevistas de pesquisa podem gerar nas famílias.

Palavras-chave: Desenvolvimento precoce, interdisciplinaridade, autismo, risco

*1 Trabalho apresentado no V Congresso Internacional de Psicopatologia Fundamental e XI Congresso Brasileiro de Psicopatologia Fundamental, setembro/2012 em Fortaleza, $\mathrm{CE}, \mathrm{Br}$.

*2 Mestranda do Programa de Pós-Graduação em Psicologia Escolar e Desenvolvimento Humano da Universidade de São Paulo - USP (São Paulo, SP, Br).

${ }^{* 3}$ Universidade de São Paulo - USP (São Paulo, SP, Br). 


\section{Introdução}

A investigação em torno da detecção de sinais iniciais de problemas de desenvolvimento, articulada ao campo da psicanálise com bebês, se constituiu como área de interesse de diversos pesquisadores nos últimos dez anos. Profissionais passaram a propor cada vez mais estudos nesta área, pois intervenções que ocorrem durante o período de instalação de um sintoma são mais favoráveis para a reversão ou diminuição de efeitos nocivos para o desenvolvimento do que aquelas que incidem após tal instalação (Golse, 2005; Teperman, 2005; Lincht, 2006; Muratori \& Maestro 2007; Bernardino, 2008; Lerner \& Kupfer, 2008; Kupfer et al. 2009; Mariotto, 2009; Laznik, 2011; Lerner, 2011).

A Pesquisa multicêntrica de indicadores clínicos de risco para o desenvolvimento infantil propôs um instrumento (Indicadores de Risco para o Desenvolvimento Infantil - IRDI) composto por 31 indicadores clínicos de desenvolvimento cuja ausência está associada a problemas em crianças aos três anos (Kupfer et al., 2008; 2009). Fundamenta-se na articulação da psicanálise com outros campos e seu uso é indicado para profissionais que atuam em dispositivos de atenção primária, razão pela qual é inespecífico para diagnóstico. O IRDI foi construído a partir de quatro eixos fundamentais em torno dos quais se organiza a subjetividade (Kupfer, 2009; Mariotto, 2009; Pesaro, 2010). Tais eixos esclarecem de que forma o laço constitutivo se enoda e como isso pode ser verificado entre os bebês e seus cuidadores. A originalidade do protocolo reside em avaliar o desenvolvimento a partir do vínculo estabelecido entre os pais e o bebê.

Dentre os transtornos da primeira infância, os quadros que podem levar a incapacitações das mais severas são aqueles pertencentes ao espectro de autismo que atualmente é considerado como uma síndrome neuropsicodesenvolvimental. Segundo Golse (2005), o autismo é fruto de um processo progressivo e multifatorial. Os estudos recentes empregam o termo autismo para se referir a um espectro de síndromes com características em comum: Transtornos Invasivos do Desenvolvimento, (DSM IV), Transtornos Globais do Desenvolvimento, (DSM IV-TR) e Transtornos do Espectro Autista, conforme autores na literatura especializada (Lampreia, 2003; Teixeira et al., 2010). As principais características do espectro são: dificuldades na interação social, déficits na comunicação, padrões restritos e repetitivos de comportamentos, interesses e atividades. Porém, por se tratar de um quadro heterogêneo, nem todas as características se manifestam concomitantemente ou estão presentes. Os sintomas mais comuns não se expressam do mesmo modo nas crianças, apesar de apresentarem certa regularidade que permite a construção de diagnósticos. 


\section{ARTIGOS}

A Pesquisa multicêntrica estabeleceu a capacidade do IRDI em detectar riscos de transtornos de desenvolvimento psíquico e atualmente está em curso um estudo ${ }^{1}$ que busca verificar se este instrumento seria útil para detectar sinais de autismo em crianças de até 18 meses. O trabalho ora proposto está inserido no contexto desta segunda pesquisa.

Para avaliar tal utilidade do instrumento, foi feita uma parceria com sete Centros de Atenção Psicossocial Infantil do Estado de São Paulo (CAPSi) a fim de investigar os irmãos (bebês) de crianças diagnosticadas como autistas. Esta medida se justifica uma vez que esses bebês são considerados de risco para autismo segundo a literatura especializada (Mecca, 2011; De Araújo, 2010; Osborne, McHugh, Saunders \& Reed, 2008; Benson \& Karlof, 2008; Yirmiya, 2006; Gorwood \& Ramoz, 2005). Vale destacar que é preciso estar atento para não aprisionar estas crianças em uma predição autística.

O presente trabalho é um desdobramento da pesquisa de detecção de sinais iniciais de autismo e discute, a partir do uso do Protocolo IRDI, como a relação pais-bebê está se desenvolvendo após a experiência com um primeiro filho autista. Será dada ênfase aos aspectos de necessidade e demanda expressos pelo bebê e decodificados pelo adulto cuidador, tomando como exemplo o momento da alimentação.

Winnicott (1958/2000) dá atenção especial à ingestão de alimentos para a construção da realidade interna do bebê ao afirmar que as fantasias do recém-nascido são emocionalmente ricas e que estas não se referem apenas ao ambiente externo, mas também ao inter-relacionamento de pessoas que vão sendo incorporadas, primeiramente, em conjunto com a ingestão de alimentos e, mais tarde, como um processo independente.

Crespin (2004) destaca que o bebê passa de sujeito da necessidade para sujeito de desejo quando o cuidador compreende que a necessidade pode ser a manifestação de um desejo a ser satisfeito. A mudança de necessidade para desejo opera em todos os registros da pulsão que, no primeiro ano de vida, são respectivamente: a oralidade (que remete ao estatuto simbólico das trocas alimentares), a especularidade e a pulsão invocante. Ainda segundo a autora, pais de crianças que se organizam de modo autístico se apresentam em estado de sideração, o que dificulta o surgimento da aptidão de recortar e atribuir sentido às expressões da criança.

Laznik (2000) afirma que é necessário separar o conceito de pulsão daquilo que seria o instinto de sobrevivência e que o cuidador deve ser capaz de entender os

${ }^{1}$ Grupo de pesquisa Transtornos do espectro de autismo: detecção de sinais iniciais e intervenção registrado no CNPq e coordenado pelo Prof. Dr. Rogério Lerner. O grupo é constituído por Ana Silvia de Morais, Andrea Tocchio, Angela di Paolo, Gabriela de Araújo, Márcia Bronzatto e Nathalia Campana. 
sinais expressos pelo bebê, ou seja, entender o que ele ainda não disse e ver o que ele ainda não é, pois "o corpo de um bebê não se reduz ao seu organismo, mas é uma construção feita da união deste organismo com algo que não vem do bebê, mas sim do Outro" (p. 67).

Partindo destas considerações teóricas, proponho a discussão do material de pesquisa de dois bebês que foram avaliados junto de suas mães com os instrumentos IRDI e MCHAT (Modified Checklist for Autism in Toddlers) - instrumento desenvolvido com a finalidade de identificar crianças com autismo a partir dos 18 meses de vida. Apresenta 23 questões que abordam temas referentes a relacionamento social, atenção conjunta, levar objetos para mostrar aos pais e capacidade de resposta da criança quando chamada por outra pessoa. Cada questão vale 1 ponto, de modo que a pontuação varia de 0 a 23 e o escore total é calculado a partir da soma dos pontos. Se a pessoa obtiver mais de 3 pontos oriundos de quaisquer dos itens, é considerada em risco para autismo; se obtiver 2 pontos derivados de itens críticos (que são as questões 2, 7, 9, 13, 14 e 15) também é considerada risco para autismo, prescindindo dos demais pontos.

\section{Vinhetas clínicas}

\section{Veridiana}

Veridiana (18 meses) é a irmã mais nova de Pedro (dez anos) e João (quatro anos). Pedro é atendido em um Centro de Atenção Psicossocial da Infância (CAPSi) há quatro anos. As três crianças estavam presentes com a mãe, Conceição, nos dias de avaliação.

Conceição contou que quando engravidou de Veridiana se sentiu fraca, com enjoos fortes e foi informada pelo médico que estava doente. Por isso, achava que logo morreria. Nesta ocasião perdeu oito quilos e pediu que a avó das crianças assumisse os cuidados com João, pois se sentia incapaz de cuidar dos filhos: eu não cuidava deles, uma vizinha trazia iogurte, deixava na geladeira e as crianças pegavam, eu não levantava da cama para nada - afirmou Conceição. A gravidez só foi confirmada no quarto mês de gestação.

A mãe disse que se sente sobrecarregada em seu dia a dia, pois Pedro e Veridiana demandam muitos cuidados. A pesquisadora pode sentir essa sobrecarga em sua própria pele, pois ao mesmo tempo em que estava atenta ao discurso de Conceição, era chamada pelas crianças para interagir com elas. As entrevistas ocorreram em meio a grande barulho. Quem mais gritava era João que ora imitava os comportamentos estereotipados de Pedro, ora as ações de Veridiana. Reclamava 


\section{ARTIGOS}

atenção para si e disse que não gostava de ter sido apelidado de chorão por seus pais: eu choro porque papai e mamãe nunca querem fazer o que eu quero. Diante disso, Conceição se limitava a repreender o filho pelo barulho que fazia.

Veridiana estava brincando com material gráfico quando sua mãe lhe ofereceu uma mamadeira. A pesquisadora perguntou para Conceição o porquê da oferta naquele momento e ela respondeu: ah, não sei... é que está no horário! Esta situação favoreceu que a pesquisadora pudesse conversar a respeito do Indicador 7 (a criança utiliza sinais diferentes para expressar suas diferentes necessidades) e do Indicador 12 (a mãe ou cuidador dá suporte às iniciativas da criança sem poupar-lhe o esforço). Conceição disse que sua bebê nunca precisou expressar suas necessidades, pois ela sempre se antecipou a tudo: eu estipulava os horários e pronto. A mãe também relatou que até hoje não deixa sua filha se esforçar para conseguir o que quer: facilito tudo, dou tudo na mão, ela aponta e eu pego.

Apesar de os momentos de alimentação de Veridiana serem movidos por horários preestabelecidos pela mãe, e não pela demonstração de desejo da bebê, foi possível observar que enquanto mama, Veridiana olha para a mãe e recebe em troca sorrisos, expressões de carinho e esta experiência parece gratificante para a dupla. Os demais indicadores do IRDI estavam presentes e a aplicação do MCHAT não apontou escore para autismo. Tudo indica que neste momento, apesar da vulnerabilidade, Veridiana está se desenvolvendo bem.

Por outro lado, as preocupações da pesquisadora se voltaram para João, que apresentou sinais de dificuldade para lidar com o fato de ter dois irmãos que exigem muitos cuidados maternos: apresentou alguns comportamentos regressivos como imitar o irmão autista e a irmã bebê, seus desenhos revelaram grau significativo de sofrimento e angústia, e ele chegou a se colocar em risco durante a aplicação dos instrumentos de pesquisa ao se pendurar na janela para chamar a atenção da mãe para si. Ao contrário de Veridiana, que não precisa expressar suas necessidades para receber o que deseja, João grita para expressar seu desejo, mas não é atendido.

Esta situação pode ser discutida tanto com o profissional de referência da família no CAPSi, quanto com a própria família. Conceição disse que se sentiu beneficiada pela oportunidade de conversar a respeito dos filhos e de sua maternidade e solicitou realizar atendimento psicoterapêutico de casal para que pudesse seguir discutindo com o marido as questões referentes à parentalidade.

\section{Marco}

Marco (18 meses) é o irmão mais novo de Juliano (oito anos) e Vinícius (seis anos). Juliano foi diagnosticado como autista e está em tratamento em um CAPSi. Apenas a mãe, Berenice, e Marco estavam presentes nas entrevistas de avaliação. 
Berenice contou que achava Juliano um bebê com desenvolvimento normal ao longo do primeiro ano de vida, mas que depois ele se isolou e não solicitava o que queria: ele podia ficar um dia inteiro sem comer que não pedia comida. Em relação a Marco, a mãe descreveu um processo de reconhecimento e estabelecimento de vínculo que foi se construindo ao longo do tempo e enfatizou que ele sempre foi um bebê risonho e que olhava para ela.

Enquanto a mãe conversava com a pesquisadora, Marco ficou explorando a sala. Era um espaço pequeno, sem atrativos, mas ele abriu sozinho todas as gavetas do armário que estavam ao seu alcance, pegou papel, caneta, um gibi e ficou brincando tranquilamente, sem solicitar a atenção da mãe ou da pesquisadora que então se dirigiu a ele dizendo: Marco você arrumou um jeito de brincar e sozinho descobriu e pegou o que tinha aqui. O bebê olhou para a pesquisadora e sorriu.

Narrar o que estava ocorrendo na aplicação favoreceu para que a mãe pudesse reconhecer que Marco é extremamente independente e não busca o olhar de aprovação do adulto para fazer o que quer (IRDI 21): se ele quer água, arrasta um banquinho até o filtro e pega sozinho, eu já disse que não pode porque é perigoso, mas ele continua fazendo! Neste momento, Marco para de riscar o papel por um instante e olha para sua mãe. A pesquisadora pergunta para Berenice por que ela acha que o filho se comporta desta forma e ela responde que não sabe ao certo, mas imagina que ele aprendeu a ser independente. Também foi perguntado à mãe como Marco pedia o queria quando tinha, aproximadamente, seis meses e ela responde: ele não pedia, não precisava, pois eu nem esperava ele sentir fome para dar comida, era tudo programado, com horários.

Apesar de o momento de alimentação de Marco ser estipulado por horários, e não pelo reconhecimento de expressões de desejo do menino, as trocas alimentares foram descritas com prazer por sua mãe. Ela disse que esse era um momento só deles, em que podia estar disponível por inteiro. Fora isso, Berenice disse que estava sobrecarregada com os cuidados da casa, o tratamento de Juliano, com o outro filho, Vinícius, e com o marido. Apesar da vulnerabilidade, Marco parece estar se desenvolvendo bem e o MCHAT não apontou escore para autismo. Sua atitude independente pode ser pensada como fator de resiliência frente a um ambiente pouco disponível, e da mesma forma que soube explorar a sala e descobrir uma forma de brincar, também está descobrindo formas de aproveitar ao máximo os momentos em que o cuidador está disponível para ele.

$\mathrm{Na}$ entrevista devolutiva a pesquisadora conversou com Berenice e Marco a respeito do que observou, a mãe disse que antes da avaliação incentivava a independência de Marco, pois esta era sua maior preocupação com o filho mais velho, mas que agora era capaz de compreender que Marco ainda era um bebê, condição que o torna dependente de cuidados. 


\section{ARTIGOS}

\section{Discussão}

Em ambos os casos relatados as mães alimentavam seus bebês sem aguardar que estes manifestassem o desejo de serem alimentados, ou seja, sem fazer a leitura de uma expressão vinda por parte da criança; o que regulava o momento de comer era o horário do relógio. Apesar desta imposição que as mães fizeram para seus bebês, podemos constatar o estabelecimento de troca alimentar, pois elas relatam esse momento com afeto, descrevem situações de interação, comunicação e possibilidade de encontro com seus filhos. Assim, mesmo que o seu discurso em relação à alimentação de seus bebês seja aparentemente relacionado ao campo da necessidade, elas não são intolerantes ou insensíveis às manifestações do bebê e, assim, não estabelecem um padrão essencialmente intrusivo para se relacionar com eles.

Dado que essas mulheres inauguraram sua maternidade sendo mães de bebês que apresentam comportamentos autísticos, e estes parecem tê-las convencido de que só dependiam delas no que se referia aos cuidados físicos, podemos levantar a hipótese de que as mães aprenderam a antecipar as necessidades orgânicas, sem esperar qualquer manifestação por parte dos seus bebês. Ser mãe de um primeiro filho autista deixou marcas na função materna destas mulheres, marcas que se refletem na relação que estabelecem com seus outros filhos. Porém, não perderam a capacidade de investir em seus bebês e de responder quando convocadas.

No primeiro caso relatado, a mãe refere ter se dado conta de que não estava em condições de cuidar de seus dois filhos mais velhos quando parou de alimentá-los, estabelecendo em sua fala uma relação importante entre alimento e cuidado - disponibilidade emocional. Conceição apresentava maior dificuldade para se relacionar com o filho do meio, porém quando essa questão pôde ser nomeada nas entrevistas de avaliação, a mãe construiu uma demanda de atendimento e João ficou mais tranquilo ao ter seu sentimento reconhecido e nomeado. Assim, partindo do pedido da mãe, a oferta de um espaço psicoterapêutico para que os pais possam ser acolhidos e seguir trabalhando as questões da parentalidade se configura como fundamental e pode ter caráter preventivo no contexto desta família.

No segundo caso, Berenice também enfatizou a importância da alimentação na relação pais-bebê quando falou da angústia que sentia quando percebia que seu filho mais velho podia ficar um dia sem comer sem manifestar qualquer tipo de reação frente a isso. Berenice estava sobrecarregada com os cuidados dedicados aos filhos, mas pareceu aproveitar-se das pequenas intervenções feitas durante a avaliação e não solicitou, nem foi constatada alguma situação que justificasse um encaminhamento além do que já recebia no CAPSi (o atendimento de Juliano e a participação no grupo de pais).

A aplicação dos instrumentos IRDI e MCHAT, associada às observações qualitativas feitas pela pesquisadora, sugerem que neste momento os dois bebês não 
apresentam risco para autismo e possuem recursos internos para lidar com as adversidades que se impõem, sejam vindas do ambiente ou do próprio organismo. Ambos são bebês extremamente vorazes: sugam rapidamente a mamadeira, tiram da sala tudo o que podem, exploram os objetos e se apoderam deles sem hesitar.

Winnicott (1958/2000) afirma que os bebês são capazes de perceber as pessoas por meio do olhar, de sentir estados de espírito, notar aprovação ou desaprovação e perceber que devido à voracidade de seu amor estão privando outros de coisas boas. $\mathrm{O}$ autor discute que o interjogo entre a voracidade do bebê e as diversas maneiras pelas quais ele irá controlar ou contrabalançar seus efeitos por meio da reconstrução ou da reparação se coloca como uma questão complexa. Assim, mesmo que a avaliação realizada não tenha constatado risco para patologias graves do desenvolvimento, é pertinente que essas crianças possam ser acolhidas e escutadas.

Tanto os efeitos que foram produzidos nas famílias ao longo das avaliações quanto a discussão realizada com a equipe do CAPSi indicam que esses "sujeitos de pesquisa" se sentiram recompensados por participar do estudo. Nesse sentido, Jimenez (2007) discute que a pesquisa pode indicar formas de intervenção que possibilitem mudança terapêutica e, para isso, os estudos devem apresentar tanto as características do paciente quanto da relação que estabelecem com o analista (pesquisador). Reid (1998) afirma que embora o diagnóstico psiquiátrico feito por questionário possa confirmar a presença e o grau de severidade do autismo, pouco informa a respeito da experiência da criança e de sua família. Desta forma, o diagnóstico é apenas uma parte da avaliação.

Além dos instrumentos padronizados, o profissional que estiver à frente das avaliações deve contar com seus recursos para identificar os sentimentos que lhes são despertados ao longo das entrevistas e utilizá-los como ferramenta para compreender a situação pesquisada. Esta ideia está em ressonância com a proposta de Salomonsson (2007) a respeito do atendimento com pais e bebês quando destaca que a contratransferência é fundamental para o processo de entendimento do analista (pesquisador). O autor afirma ainda que quando o tema da pesquisa é a vida do bebê devemos ir além da contratransferência e recorrer aos conhecimentos produzidos pela psicologia do desenvolvimento e pela semiótica para poder formular uma compreensão clínica.

Nos dois casos relatados a pesquisadora utilizou as emoções que foram despertadas no "aqui agora" das entrevistas para formular questões que facilitaram a emergência dos conteúdos emocionais de cada família e, desta forma, a compreensão clínica de cada caso. No primeiro caso, foi fundamental que a pesquisadora sentisse a sobrecarga e a falta de espaço, de continência, para os conteúdos emocionais. No segundo caso, sentir que não estava dando atenção ao bebê que explorava a sala por conta própria contribuiu para que a pesquisadora se aproximasse da experiência emocional daquela dupla mãe-bebê. 


\section{ARTIGOS}

\section{Considerações finais}

O pesquisador parte de noções já estabelecidas que justifiquem sua inserção quando determina o campo de pesquisa para realizar seu estudo, mas é somente quando se lança no campo e encontra com seus sujeitos que as questões vão se impondo e se construindo.

Nos dois casos relatados podemos encontrar fatores de risco conhecidos na literatura que justificariam o encaminhamento para atendimento dos pais e dos bebês, porém a proposta é que seja oferecido para as famílias um período de avaliação, para que questões particulares possam emergir, propiciando que sejam formuladas demandas de atendimento de acordo com as suas necessidades. A aplicação do IRDI vem se mostrando como promissora no que se refere a criar uma atmosfera de acolhimento e escuta para as famílias, norteada por questões referentes ao desenvolvimento inicial.

As entrevistas de avaliação ajudaram na investigação da relação que se estabeleceu entre as mães e seus bebês durante a troca alimentar e quais foram os possíveis efeitos em decorrência. É possível se pensar, diante dos dados coletados, que os bebês aprenderam a aproveitar ao máximo os momentos de disponibilidade da mãe para com eles, contribuindo, dessa forma, para a sua voracidade na relação com os objetos.

Mães de crianças autistas não perdem sua capacidade de reconhecer manifestações de desejo em seus filhos mais novos e não precisam ficar aprisionadas no registro da necessidade orgânica. Porém, essa capacidade de leitura das mães pode se encontrar adormecida, o que demanda um espaço de escuta para que possam recuperar a confiança abalada e estabelecer novas formas de relacionamento com os filhos. Da mesma forma, os irmãos não estão fadados a apresentar risco de desenvolvimento em decorrência de vulnerabilidades orgânicas e ambientais. $\mathrm{O}$ encontro com essas crianças sugere que elas podem se beneficiar de um espaço de acolhimento que não tenha necessariamente fins de tratamento psicoterapêutico, sugere também que o espaço desses bebês na família pode ser beneficiado se seus pais forem ajudados nas questões referentes à parentalidade.

Agradecimentos: Os autores agradecem à Fundação de Amparo à Pesquisa do Estado de São Paulo e à Fundação Maria Cecília Souto Vidigal, pelo financiamento da pesquisa.

\section{Referências}

Benson, P. R. \& Karlof, K. L. (2008). Child, parent, and family predictors of latter adjustment in siblings of children with autism. Research in Autism Spectrum Disorders, 2, 583-600. 
Bernardino, L. M. F. (2008). A avaliação psicanalítica das crianças da Pesquisa Multicêntrica de Indicadores Clínicos para o desenvolvimento infantil: impasses teóricos e clínicos. In: L. M. Atem (Org.). Cuidados no início da vida: clínica, instituição, pesquisa e metodologia (pp. 123-128). São Paulo: Casa do Psicólogo.

Crespin, G. C. (2004). A clínica precoce: o nascimento do humano. São Paulo: Casa do Psicólogo.

De Araujo, G. X. (2010). Quando não há mais espaço: um estudo sobre a frátria das crianças autistas. Estilos da Clínica, São Paulo, 15(1), 126-143.

Golse, B. (2005). Autismo infantil: despiste e prevenção. Rev. latinoam. psicopatol. fundam, 8(3), 428-442. Disponível em: <http:/www.fundamentalpsychopathology.org/uploads/ files/revistas/volume08/n3/autismo_infantil_despiste_e_prevencao.pdf $>$. Acesso em: 11 mar. 2013.

Gorwood, P. \& Ramoz, N. (2005). Facteurs génétiques dans l'autisme. In B. Golse, \& P. Delion (Orgs.). Autisme: état des lieux et horizons (pp. 71-88). Paris: Érès.

Jimenez, J. P. (2007). Can research influence clinical practice? International Journal of Psychoanalysis, 88, 661-679.

Kupfer, M. C. M. et al. (2010). Valor preditivo de indicadores clínicos de risco para o desenvolvimento infantil: um estudo a partir da teoria psicanalítica. Rev. Latinoam. Psicopatol. Fundam., São Paulo, 13(1), 31-52. Disponível em: <http://www.scielo.br/ scielo.php?script=sci_arttext\&pid=S1415-47142010000100003 \&lng=en\&nrm=iso>. Acesso em: 28 fev. 2013 .

Lampreia, C. (2003). Avaliações quantitativa e qualitativa de um menino autista: uma análise crítica. Psicologia em Estudo, Maringá, 8(1), 57-65.

Laznik, M. C. (2000). Psicanalistas que trabalham em saúde pública. Pulsional Revista de Psicanálise, São Paulo, 13(132), 62-78.

Laznik, M. C. (2011). Rumo à fala. Rio de Janeiro: Companhia de Freud.

Lerner, R. (2011). Indicadores Clínicos de Risco para Desenvolvimento Infantil - IRDI: verificação da capacidade discriminativa entre autismo, retardo mental e normalidade. Tese de Livre-docência. Instituto de Psicologia da Universidade de São Paulo, São Paulo.

Lerner, R. \& Kupfer, M. C. M. (Orgs.). (2008). Psicanálise com crianças: clínica e pesquisa. São Paulo: Escuta/Fapesp.

Lincht, R. W. (2006). Na sala de espera esperando o Outro: uma interlocução entre as pesquisas neurológicas e a psicanálise. In: L M. F. Bernardino (Org.). O que a psicanálise pode ensinar sobre a criança, sujeito em constituição. São Paulo: Escuta.

Mariotto, R. M. M. (2009). Cuidar, educar e prevenir: as funções da creche na subjetivação de bebês. São Paulo: Escuta.

Mecca, T. P. et al. (2011). Rastreamento de sinais e sintomas de transtornos do espectro do autismo em irmãos. Revista de Psiquiatria do Rio Grande do Sul-APRS, 33(2), 116-120. 


\section{ARTIGOS}

Muratori, F. \& Maestro, S. (2007). Early signs of autism in the first year of life. In S. Acquarone (Org.). Signs of autism in infants: recognition and early intervention (pp. 46-61). Londres: Karnac Books.

Osborne, L. A., McHugh, L., Saunders, J. \& Reed, P. (2008). The effect of parenting behaviors on subsequent child behavior problems in autistic spectrum conditions. Research. Autism Spectrum Disorders, 2, 249-263.

Pesaro, M.E. (2010). Alcance e limites teórico-metodológicos da pesquisa multicêntrica de indicadores clínicos de risco para o desenvolvimento infantil. Tese de Doutorado. Instituto de Psicologia da Universidade de São Paulo, São Paulo.

Reid, S. (1998). The assessment of the child with autism. In A. Alvarez, \& S. Reid (Orgs.). Autism and Personality: findings from the Tavistock autism workshop. Inglaterra: Routledge.

Salomonsson, B. (2007). Talk to me baby, tell me what's the matter now. Semiotic and developmental perspectives on communication in psychoanalytic infant treatment. International Journal of Psychoanalysis, 88, 127-146.

Teixeira, M. C. T. V. et al. (2010). Literatura científica brasileira sobre transtornos do espectro autista. Rev. Assoc. Med. Bras, 56(5), 607-614.

Teperman, D. W. (2005). Clínica psicanalítica com bebês: uma intervenção a tempo. São Paulo: Casa do Psicólogo/Fapesp.

Winnicott, D. W. (2000). Da pediatria à psicanálise. Rio de Janeiro: Imago. (Trabalho original publicado em 1958).

Yirmiya, N. et al. (2006). The development of siblings of children with autism at 4 and 14 months: social engagement, communication, and cognition. Journal of Child Psychology and Psychiatry, 47(5), 511-523.

\section{Resumos}

(Exchanges of food among siblings of autistic babies and their mothers:

Risk or resource?)

Based on the fact that food intake is significant for the construction of a baby's internal reality, the present article discusses the relationship that mothers - whose oldest child was diagnosed with autism - established with their younger children at meal time. Clinical vignettes are presented to call attention to the concepts of need and demand, aspects of the relationship between parents and children, and the therapeutic effects that the research interviews may bring about in the families.

Key words: Early development, interdisciplinarity, autism, risk 
(Echanges de nourriture entre bébés frères et sœurs d'autistes et de leurs mères: risque ou ressource?)

En prenant comme point de départ l'importance de l'ingestion des aliments pour la construction de la réalité interne du bébé, cet article vise à discuter le rapport que les mères de premiers-nés autistes établissent avec leurs autres enfants pendant leur alimentation. Nous présentons des vignettes cliniques qui mettent l'accent sur les notions de besoin et de demande, les aspects du rapport entre les parents et l'enfant et les effets thérapeutiques qui peuvent résulter des entretiens de la recherche pour les familles.

Mots-clés: Développement précoce, interdisciplinarité, autisme, risque

(Intercambios de alimentación entre bebés hermanos de niños con autismo y sus madres: riesgo o recurso?)

Asumiendo la importancia que la ingesta de alimentos tiene para construir la realidad interna del bebé, este estudio discute la relación que las madres del primer hijo diagnosticado como autista establecen con sus hijos pequeños durante la alimentación. Se presentaran viñetas clinicas enfatizando las nociones de necesidad $y$ de demanda, en las relaciones entre padres y niños y los efectos terapéuticos que pueden generar las entrevistas de investigación en las familias.

Palabras clave: Desarrollo temprano, interdisciplinaridad, autismo, riesgo

(Nahrungsaustausch zwischen Geschwistern von autistischen Kleinkindern und ihren Müttern: Risiko oder Möglichkeit?)

Ausgangspunkt ist die Notwendigkeit, sich zu ernähren und was dies für die Gestaltung der Inneren Realität eines Kleinkindes bedeutet. Auf dieser Grundlage diskutiert diese Untersuchung die Beziehung, die Mütter, deren erstes Kind autistisch ist, zu den jüngeren Geschwistern während der Ernährung aufbauen. Es werden einige klinische Kurzvideos gezeigt, die Aspekte der Notwendigkeit und des Bedürfnisses hervorheben, Aspekte der Eltern-Kind-Beziehung und mögliche therapeutische Auswirkungen der Forschungsinterviews für Familien.

Schlüsselwörter: Frühreife Entwicklung, Interdisziplinarität, Autismus, Risiko

Citação/Citation: Campana, N. T. C. \& Lerner, R. (2014, junho). Trocas alimentares entre bebês irmãos de autistas e suas mães: risco ou recurso? Revista Latinoamericana de Psicopatologia Fundamental, 17(2), 191-203.

Editor do artigo/Editor: Manoel Tosta Berlinck 


\section{ARTIGOS}

Recebido/Received: 8.4.2013/4.8.2013 Aceito/Accepted: 27.11.2013/11.27.2013

Copyright: (C) 2009 Associação Universitária de Pesquisa em Psicopatologia Fundamental/ University Association for Research in Fundamental Psychopathology. Este é um artigo de livre acesso, que permite uso irrestrito, distribuição e reprodução em qualquer meio, desde que o autor e a fonte sejam citados / This is an open-access article, which permits unrestricted use, distribution, and reproduction in any medium, provided the original authors and sources are credited.

Financiamento/Funding: A pesquisa foi financiada pela Fundação de Amparo à Pesquisa do Estado de São Paulo - Fapesp e pela Fundação Maria Cecília Souto Vidigal / The research was funded by the Fundação de Amparo à Pesquisa do Estado de São Paulo - Fapesp and by the Fundação Maria Cecília Souto Vidigal

Conflito de interesses/Conflict of interest: Os autores declaram que não há conflito de interesses / The authors declare that has no conflict of interest.

\section{Nathalia Teixeira Caldas Campana}

Psicóloga graduada pela Pontifícia Universidade Católica de São Paulo - PUC-SP (São Paulo, SP, Br); Especialização em Psicologia da Infância pelo Depto. de Pediatria da Universidade Federal de São Paulo - Unifesp (São Paulo, SP, Br); Mestre em Psicologia Escolar e Desenvolvimento Humano da Universidade de São Paulo - USP (São Paulo, SP, Br).

Av. Macuco, 726/1602

04523-001 São Paulo, SP, Br

e-mail:nacampana@gmail.com

\section{ROGÉRIO LERNER}

Psicanalista; Professor Associado do Instituto de Psicologia da Universidade de São Paulo IPUSP (São Paulo, SP, Br); Doutor e Livre-Docente em Psicologia pelo mesmo Instituto; Pós-doutorado pela Universidade Pierre et Marie Curie (Paris, Fr); Fellow do College of Research Training Programme University College London/International Psychoanalytical Association; Membro do Núcleo de Apoio à Pesquisa em Neurodesenvolvimento e Saúde Mental; Lider do grupo de pesquisa do CNPq intitulado "Transtornos do espectro de autismo: detecção de sinais iniciais e intervenção".

Av. Prof. Mello Moraes, 1721 - Cidade Universitária

05508-030 São Paulo, SP, Br

e-mail: rogerlerner@usp.br / rogerlerner@uol.com.br 